\title{
Isolation of CaSLN1 and CaNIK1, the genes for osmosensing histidine kinase homologues, from the pathogenic fungus Candida albicans
}

\author{
Shigehisa Nagahashi, ${ }^{1,2}$ Toshiyuki Mio, ${ }^{2}$ Naomi Ono, ${ }^{2}$ \\ Toshiko Yamada-Okabe, ${ }^{3}$ Mikio Arisawa, ${ }^{2}$ Howard Bussey ${ }^{1}$ \\ and Hisafumi Yamada-Okabe ${ }^{2}$
}

Author for correspondence: Hisafumi Yamada-Okabe. Tel: +81 46747 2242. Fax: +81467465320. e-mail: hisafumi.okabe@roche.com

\begin{abstract}
1 Department of Biology, McGill University, 1205 Dr. Penfieid, Montreal, Quebec, Canada H3A 1B1

2 Department of Mycology, Nippon Roche Research Center, 200 Kajiwara, Kamakura, Kanagawa 247, Japan

3 Department of Hygiene, School of Medicine, Yokohama City University, 3-9, Fukuura, Kanazawaku, Yokohama 236, Japan
\end{abstract}

\begin{abstract}
Recent studies have revealed that fungi possess a mechanism similar to bacterial two-component systems to respond to extracellular changes in osmolarity. In Saccharomyces cerevisiae, $\operatorname{SIn1p}$ contains both histidine kinase and receiver (response regulator) domains and acts as an osmosensor protein that regulates the downstream HOG1 MAP kinase cascade. SLN1 of Candida albicans was functionally cloned using an S. cerevisiae strain in which SLN1 expression was conditionally suppressed. Deletion analysis of the cloned gene demonstrated that the receiver domain of $C$. albicans SIn 1p was not necessary to rescue SLN1-deficient S. cerevisiae strains. Unlike $S$. cerevisiae, a null mutation of $C$. albicans SLN1 was viable under regular and high osmotic conditions, but it caused a slight growth retardation at high osmolarity. Southern blotting with C. albicans SLN1 revealed the presence of related genes, one of which is highly homologous to the NIK1 gene of Neurospora crassa. Thus, C. albicans harbours both SLN1- and NIK1-type histidine kinases.
\end{abstract}

Keywords: Candida albicans, cloning, osmosensor, two-component system, histidine kinase

\section{INTRODUCTION}

Two-component systems which involve a phosphorelay from the histidine of the sensor kinase to the aspartic acid of the response regulator are widespread in bacteria (Bourret et al., 1991; Stock et al., 1991; Parkinson \& Kofoid, 1992). Regulatory proteins similar to bacterial two-component systems are also found in some eukaryotes (Brown et al., 1993; Ota \& Varshavsky, 1993; Alex et al., 1996; Chang et al., 1993; Hua et al., 1995; Wilkinson et al., 1995). In Saccharomyces cerevisiae, $\operatorname{Sln} 1 \mathrm{p}$ consists of an extracellular sensor, a kinase and a receiver domain (Ota \& Varshavsky, 1993; Maeda et al., 1994) and acts as an osmosensor protein (Maeda et al., 1994). Under low osmolarity conditions, a specific histidine in the kinase domain is autophosphorylated. The phosphate moiety of this histidine is first transferred to a certain aspartic

Abbreviation: 5-FOA, 5-fluoroorotic acid.

The GenBank/EMBL/DDBJ accession numbers for the sequences reported in this paper are AB006362 (SLN1) and AB006363 (NIK1). acid within the receiver domain and then via a phosphorelay to the downstream proteins Ypd1p and Ssk1p, leading to the shut off of the HOG1 MAP kinase cascade (Brewster et al., 1993; Maeda et al., 1994; Posas et al., 1996). Histidine kinase activity and phosphorylation of $\operatorname{Sln} 1 \mathrm{p}$ are essential for growth at low osmolarity because a mutation of either the autophosphorylating histidine or the receiver aspartic acid of $S \ln 1 \mathrm{p}$ is lethal under these conditions (Maeda et al., 1994). Increased osmolarity hampers the histidine kinase activity of $\operatorname{Sn} 1 \mathrm{p}$, which in turn activates downstream HOG1 MAP kinase (Brewster et al., 1993; Maeda et al., 1994; Posas et al., 1996).

Involvement of a histidine kinase in an osmosensing pathway has also been reported in Neurospora crassa (Alex et al., 1996). The predicted product of the $N$. crassa NIK1 gene possesses two domains that are related to sensor histidine kinases and response regulators of bacterial two-component proteins (Alex et al., 1996). Nik1p is an apparent cytoplasmic protein with six repeats of about 90 aa that may form a coiled-coil structure. Deletion of the NIK1 gene caused aberrant 
hyphal morphology, a phenotype more prominent under high osmotic conditions (Alex et al., 1996).

To address whether a histidine kinase osmosensing mechanism is conserved in other yeasts, we attempted the functional cloning of Candida albicans SLN1. In addition, probing a C. albicans genomic library with the C. albicans SLN1 gene identified a gene that is highly related to NIK1 of $N$. crassa. Thus, it seems that $C$. albicans has both Sln1p- and Nik1p-type histidine kinases which may allow adaptation to different osmotic conditions.

\section{METHODS}

Plasmid construction and yeast strain. A $700 \mathrm{bp}$ HindIII-HindIII fragment that harbours the tetO-HOP1 chimaeric promoter, UAS and URS, was excised from $\mathrm{p} 97 \mathrm{t}$ (Nagahashi et al., 1997), ligated with the DNA fragment containing the hisG-URA3-hisG module isolated from pMPY-ZAP (Schneider et al., 1996) and cloned into Bluescript SKII + to generate $\mathrm{p} 97 \mathrm{tZAP}$. Replacement of the cognate SLN1 promoter with a tetracycline-controllable promoter (Nagahashi et al., 1997) was achieved by the one-step gene replacement method (Baudin et al., 1993; Schneider et al., 1996) with slight modification. DNA fragments harbouring target sequences of the $S L N 1$ and tetracycline-regulated promoters and the his $G-U R A 3-h i s G$ module were amplified by PCR using p97tZAP as a template and a pair of primers, $5^{\prime}$ CATCGAAAACAGCACGAACAAAAGCCAACTCAC'TACATTTTAGAACAGCTATGACCATG 3' and 5' TCCAATTTTGATGGCAGGCCAAATCGCATTTGTATTGGAATTCTTTTCTGAGATAAAG 3'. The resulting DNA fragment was transfected into an $S$. cerevisiae strain, YPH499 (MATa ade2 his3 leu2 lys 2 ura3) that had been transfected with pINFAGAL4 and which constitutively expressed the tetR-GAL4 fusion activator (Nagahashi et al., 1997). After confirming the correct integration of the tetO-HOP1 chimaeric promoter by PCR and Southern blotting, transfectants were selected by 5-fluoroorotic acid (5-FOA) and used for experiments. For convenience, we designated the above strain as Tet-SLN1. To determine the region of CaSLN1 essential for complementing ScSLN1, deletion mutants of CaSLN1 were cloned into the unique BamHI site of YEp24T (YamadaOkabe et al., 1996) and transfected into another $S$. cerevisiae strain, A451 (MAT $\alpha$ can1 leu2 trp1 ura3 aro7) in which the original SLN1 locus was disrupted by LEU2 but where episomal copies of SLN1 in pYES2 (Invitrogen) were maintained under the control of the GAL1 promoter. This strain, termed pYES-SLN1, was unable to grow in the presence of either 5-FOA or glucose as sole carbon source. Primers used to amplify $S c S L N 1$ were $5^{\prime}$ CCCGGGGAATTCATGCGATTTGGCCTGCCA $3^{\prime}$ and $5^{\prime}$ CCCGGGGAATTCTCATTTGTTATTTTTCTT 3'. For ScSLN1 disruption, the $2 \cdot 3 \mathrm{~kb}$ PstI-PstI region of ScSLN1 was replaced by LEU2.

Screening the $C$. albicans SLN1 and NIK1 genes. Tet-SLN1 cells were transfected with a $C$. albicans genomic DNA library and spread on plates containing $50 \mu \mathrm{g}$ tetracycline $\mathrm{ml}^{-1}$. After incubation at $30^{\circ} \mathrm{C}$ for $3 \mathrm{~d}$, colonies appeared on the plates, cells were collected and plasmid DNA was recovered from them. After a second screening, the essential region of the insert DNA that conferred tetracycline-resistant growth of Tet-SLN1 was determined by cloning each DNA fragment in YEp24T (Yamada-Okabe et al., 1996) and in pRS416 (Stratagene).
For cloning the NIK1 gene of C. albicans, a C. albicans genomic DNA library was screened with the $2 \cdot 1 \mathrm{~kb} K p n \mathrm{I}-$ SalI fragment of the C. albicans SLN1 gene as probe. Hybridization was carried out under low stringency conditions in a buffer containing $0.25 \mathrm{M}$ sodium phosphate $(\mathrm{pH} 7 \cdot 2), 2 \times \mathrm{SSC}$ $(1 \times$ SSC contains $150 \mathrm{mM} \mathrm{NaCl}$ and $15 \mathrm{mM}$ sodium citrate), $1 \%(\mathrm{w} / \mathrm{v})$ bovine serum albumin, $1 \mathrm{mM} \operatorname{EDTA}, 0 \cdot 1 \%(\mathrm{w} / \mathrm{v})$ SDS and $25 \%(\mathrm{w} / \mathrm{v})$ formamide at $37^{\circ} \mathrm{C}$ for $12 \mathrm{~h}$. Radiolabelling of DNA with $\left[\alpha^{32} \mathrm{P}\right] \mathrm{dCTP}$ and DNA sequencing were carried out as described by Sambrook et al. (1989). Construction of C. albicans genomic DNA library was reported previously (Yamada-Okabe et al., 1996).

Disruption of CaSLN1. The $2 \cdot 3 \mathrm{~kb} K p n \mathrm{I}-K p n \mathrm{I}$ fragment of CaSLN1 was cloned in pUC19 and the resulting plasmid digested with BalI and SnaBI followed by ligation with a $3.8 \mathrm{~kb}$ Bam HI-XbaI fragment carrying the his G-URA3-bisG module, generating pCASLN1U. Thus, the $0.6 \mathrm{~kb}$ SnaBI-BalI region of CaSLN1 was replaced by the hisG-URA3-hisG module in pCASLN1U. After pCASLN1U was linearized by digestion with $P v u \mathrm{II}, 10 \mu \mathrm{g}$ DNA was transformed into $C$. albicans CAI4 (ura3s::imm434/ura3s::imm434) cells by the lithium acetate method (Sanglard et al., 1997). Before and after a second round of transformation, the URA3 gene was excised by 5-FOA as described previously (Mio et al., 1996). Unless otherwise specified, C. albicans cells were cultured in YPD $(1 \%, \mathrm{w} / \mathrm{v}$, yeast extract; $2 \%, \mathrm{w} / \mathrm{v}$, peptone; $2 \%, \mathrm{w} / \mathrm{v}$, glucose) in the presence or absence of $1.5 \mathrm{M} \mathrm{NaCl}$.

\section{RESULTS}

\section{Functional cloning of the C. albicans SLN1 gene}

To isolate a C. albicans SLN1 gene by functional cloning, we generated an $S$. cerevisiae strain in which SLN1 expression was conditionally repressed. This strain, designated Tet-SLN1, grew normally in the absence of tetracycline, while its growth was severely impaired by the addition of $50 \mu \mathrm{g}$ tetracycline $\mathrm{ml}^{-1}$. Transfection of Tet-SLN1 with intact $S$. cerevisiae SLN1 restored normal growth even in the presence of $50 \mu \mathrm{g}$ tetracycline $\mathrm{ml}^{-1}$, demonstrating that the growth defect of Tet-SLN1 caused by tetracycline was largely due to the repression of SLN1 expression.

Tet-SLN1 cells were transfected with a C. albicans genomic DNA library that was constructed with a vector harbouring the $S$. cerevisiae TRP1 gene and a $2 \mu$ replication origin (Yamada-Okabe et al., 1996) and transfectants were selected using $50 \mu \mathrm{g}$ tetracycline $\mathrm{ml}^{-1}$. From $10^{4}$ independent colonies, three clones grew in the presence of $50 \mu \mathrm{g}$ tetracycline $\mathrm{ml}^{-1}$. The plasmid DNA was recovered from these clones and a restriction map of each insert DNA determined. Although the restriction maps of these three clones differed from each other, the map of clone 1 coincided with the pattern of a $C$. albicans genomic Southern blot that was obtained using the $S$. cerevisiae $S L N 1$ gene as a probe and this clone was analysed further.

Deletion analysis with this clone demonstrated that a $5.6 \mathrm{~kb}$ EcoRV-Xhol region was sufficient to rescue TetSLN1 cells in the presence of tetracycline (Fig. 1a). Sequencing of this region revealed that it contained a long ORF of $3.6 \mathrm{~kb}$, with a coding sequence possibly 
(a) CaSLN1
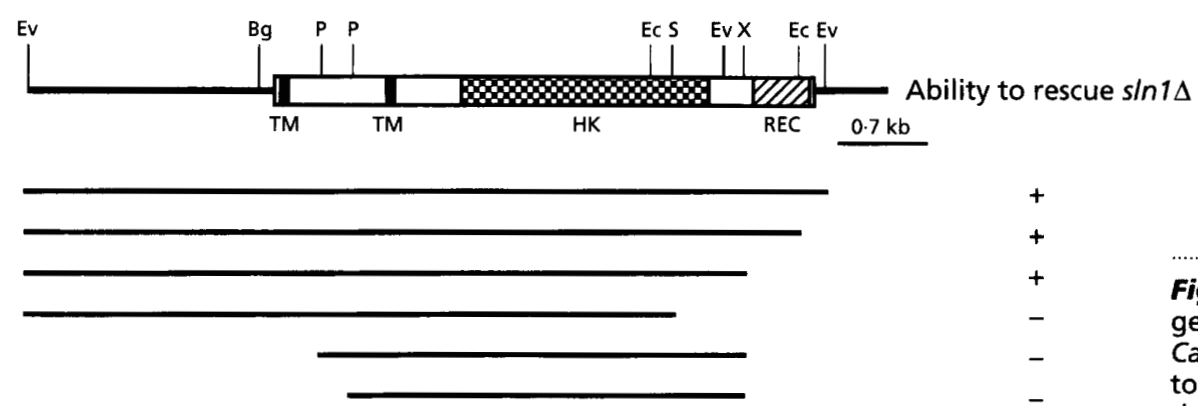

$+$

$+$

\begin{abstract}
Fig. 1. Restriction maps of C. albicans genomic DNA fragments that contain CaSLN1 (a) and CaNIK1 (b) are illustrated together with the expected structures of their products. The ability of deletion mutants to rescue pYES-SLN 1 cells $(\sin 1 \Delta)$ in the presence of 5 -FOA is indicated as ' + ' and ' - ', respectively. Ba, BamHI; $\mathrm{Bg}, B g / l$; Ec, EcoRI; Ev, EcoRV; P, Pstl; S, Sall; X, Xhol; TM, transmembrane domain; HK, histidine kinase domain; REC, receiver domain; REP, repeats of an approximately 90 aa motif.
\end{abstract}

extending further downstream. To obtain a clone containing the missing $3^{\prime}$ end of the ORF, we screened the same $C$. albicans genomic DNA library using a $0.5 \mathrm{~kb}$ Sall-Xhol fragment as a probe. Sequencing of a clone containing the complete ORF revealed that the predicted product of the gene is a $150 \mathrm{kDa}$ protein highly similar to $S$. cerevisiae $\operatorname{Sln} 1$ p (37\% identity) (Fig. 2a) and the gene was designated C. albicans SLN1 (CaSLN1). To avoid confusion, the $S$. cerevisiae $S L N 1$ was termed ScSLN1 in this study. Like ScSln1p, CaSln1p has no Nterminal signal sequence, but possesses two potential transmembrane helices in its N-terminal half (Fig. 3a). Sequence identity between $C a S \ln 1 p$ and $\mathrm{ScSln} 1 \mathrm{p}$ was remarkably high within the histidine kinase and receiver domains located in the middle of the protein and near the C-terminal end, respectively, and both the essential phosphorylating histidine at position 576 and receiver aspartic acid at position 1144 of $S c S \ln 1 \mathrm{p}$ are conserved in CaSln1p (Fig. 2a).

As described above, the initially isolated CaSLN1 clone lacked the C-terminal receiver domain, suggesting that the receiver domain of $C a S \ln 1 p$ is not crucial to complement a SCSLN1 deletion. We confirmed this by making a series of deletion mutants and transfecting them into another $S$. cerevisiae strain, pYES-SLN1. Consistent with the results of the functional cloning of CaSLN1, the $5.6 \mathrm{~kb}$ EcoRV-Xhol fragment of CaSLN1 that eliminates the receiver domain from CaSln $1 p$ still rescued pYES-SLN1 in the presence of 5-FOA or glucose, while further deletion from $\mathrm{C}$ terminus, destroying half of the probable ATP-binding site within the histidine kinase domain lead to loss of the ability to complement ScSLN1 (Figs 1a and 4).

\section{Disruption of CaSLN1}

To study the function of CaSLN1, we generated the homozygous cas $\ln 1 \Delta$ null mutant strain by means of the ura-blaster protocol (Fonzi \& Irwin, 1993). Using this strategy, the first one-third of the histidine kinase domain, including the probable autophosphorylating histidine at position 519 of $C a S \ln 1$ p, was replaced by the his $G$ sequence of Salmonella typhimurium (Fig. 5a). The correct integration of the his $G$ sequence into the expected loci was confirmed by Southern blotting (Fig. 5 b). Unlike $S$. cerevisiae, the homozygous casln $1 \Delta$ null mutant of $C$. albicans grew under both normal and high osmotic conditions and sustained an ability to form hyphae. However, the homozygous null mutation, but not the hemizygous mutation of CaSLN1 caused weak growth retardation in the presence of $1.5 \mathrm{M} \mathrm{NaCl}$ (Fig. 6a). Moreover, the homozygous cas $\ln 1 \Delta$ null mutant cells elongated in the presence of $1.5 \mathrm{M} \mathrm{NaCl}$ (Fig. 6b). Similar morphological changes of the homozygous cas $\ln 1 \Delta$ null mutants were also observed in the presence of $1 \mathrm{M}$ sorbitol or $1 \mathrm{M} \mathrm{KCl}$ (data not shown).

The above results clearly demonstrate that CaSLN1 is not an essential gene in $C$. albicans and may imply that C. albicans harbours other genes, possibly histidine kinases, to adapt to high osmolarity.

\section{Cloning the $C$. albicans $N I K 1$ gene}

In an attempt to search for other histidine kinase genes in C. albicans, we performed genomic Southern blotting with a part of CaSLN1 DNA corresponding to the histidine kinase domain of CaSln $1 p$ and detected several discrete bands that were not derived from CaSLN1 alleles. A genomic DNA library was again screened with the $2 \cdot 1 \mathrm{~kb} K p n \mathrm{I}-E c o$ RI fragment of CaSLN1 as a probe and a clone distinct from CaSLN1 was obtained. This clone contained an ORF that could encode a $119 \mathrm{kDa}$ protein highly similar to N. crassa Nik1p (50\% identity) (Fig. 2b) and the gene was termed C. albicans NIK1 (CaNIK1). Like N. crassa Nik1p, CaNik1p contains five repeats of about 90 aa within the N-terminal half (Figs $1 \mathrm{~b}$ and $2 \mathrm{~b}$ ) and a hydropathy plot of CaNik1p lacks any apparent transmembrane domain (Fig. 3b). However, 
(a)

SCSIn1p 1 : MRFGLPSKLE LTPPFRIGIR TQLTALVSTV ALGSLIILAV TAGVYFTSNY KNLRSDRLYI AAQLKSSQID QTLNYLYYQA YYLASRDALQ SSLTSYVAGN

CaSln1p $1:$ MR--------RLKIGIR PQLIIIVCFA SLFSLIILGI VTGIYFSANL KNLRLERLLV ISQLKRTQVQ QAIQYIAYQV MTVSEVDSLT VPLSNYRAGN

ScSln1p $101:$ KSADNWVDSL SVIQKFLSSS NLFYVAKVYD SSFNAVLNAT NNGTGDLIPE DVLDSLFPLS TDTPLPSSLE TIGIL--TDP VLNSTD---- -YLMSMSLPI

CaSin1p $90: \quad$ NSKAVFSEAQ NYLQYYLTT DSFTAARLYD LDLQVVASSF DNMT--LISE SAQDVVYPLQ PNRSMPPVLG TPSGLYFTGP IANNSDNFNS RYFMGITVPV

SCSInlp $194:$ FANPSIILTD SRVYGYITII MSAEGLKSVF NDTTALEHST IAIISAVY-- ------NSQ GKASGYHFV- -FPPYGSRSD LPQKVFSIKN DTFISSAFRN

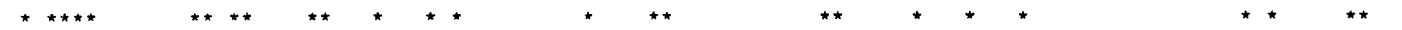

CaSln1p $188:$ LSNSSIILSQ PSISGYLTIV AAAESIRSAL NSTSEDDYQA MA-VQPVYGD POKGIDNLSQ NAYNGDNEVI GFKLVFPVEN SLLEAGTIYN INSSSSMKTA

SCSInlp $283:$ GKGGSLKQTN ILS--TRNTA LGYSPCS--F NLVNWVAIVS QPESVFLSPA TKLAKIITGT VIAIGVFVIL LTLPLAHWAV QPIVRLQKAT ELITEGRGLR

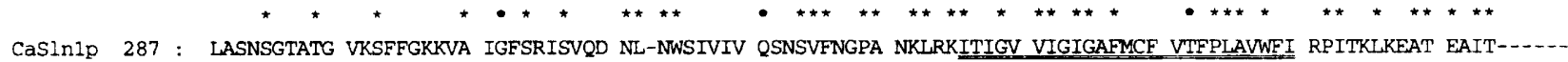

SCSln1p $379:$ PSTPRTISRA SSFKRGFSSG FAVPSLLOF NTAEAGSTTS VSGHG-GSGH GSGAAFSANS SMKSAINLGN EKMSPPEEEN KIPNNHTDAK ISMDGSLNHD CaSlnlp $380: \ldots \ldots$ ScSIn1p 478 : LUGPHSLRHN DTDRSSNRSH ILTTSANLTE ARLPDYRRLF SDELSDLTET FNTMTDALDQ HYALLEERVR ARTKQLEAAK IEAEAANEAK TVELANISHE

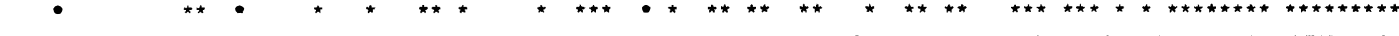
CaSln1p $428:$ TGKRNS--- --INSSSFSS SYSTGIRL-P ARIPRSKKIF KDELTELSEA FNIMTEFIDK QYTHLEDRVK LRTKELEASK IQAEAANEAK TVFIANISHE

SCSIn1p 578 : LRTPLNGILG MTAISMEETD VNKIRNSLKL IFRSGELLLH ILTELLTFSK NVLORTKLEK RDFCITDVAL QIKSIFGKVA KDQRVRLSIS LFPNLIRTMV CaSln1p $521:$ LRTPLNGILG MTSIAMEEED PNVIHDSLKL IHRSGELLLH ILTELLTYSK NTLNRSKLEK SNFQILETVY QVRSIFNKLA HDQRVNFKIL VKPNIFRKLI

SCSIn1p $678:$ LWGDSNRIIQ TVMNUSNAL KFTPVDGTVD VRMKLLGEYD KELSEKRQYK EVYI-_-

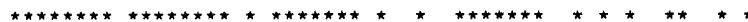

CaSln1p 621 : TYGDSNRIIQ IVMNLVSNSL KFTPVDGSVS VSFKLLGEYD HERSKKLDYK KVCILNDSSS STVAVPPPTP PSDTKPNPKP KSTPTPKPDP TRSHLVDHNN SCSln1p 732 : - ------ -KKGTEVTE NLETT---- CaSin1p 721 : RSANTTSPLT PVRKPTINQTK NKSITNNVTK QNMKIRKKKK TNKNLHNNNN NNKNDNSDFL MNRRLSGSHK FNNTNDEELS PTAIEKNIDK YLTSSADSDN SCSln1p $769:$ GSNRDTSTIQ EEIT--1 CaSln1p $821:$ ISVTTLSTVQ YETTIFESQF KSKPLPALPV DAKPQVSGKI DENDVNDEDP SGGSIKDDDS EDTINEKQGI SSSPSSSSSS NEKQENSPRS NDSTTVTVTR SCSIn1p 817 : SYDN-----A IFNS--QFNK AP-----GSD DEEGGN---- LGRPIENPKT WVISIEVEDT GPGIDPSLQE SVFHPFVQGD QTLSRQYGGT GLGLSICRQL CaSlnlp 921 : PRHIMMPSAQ DFKSYPTFDK KPEYDSNMSN NEIVKNNRVY RIRNMYQPKV WVIQIEVTDT GPGIEPALOE KVFEPFVQGD QTLSRSYGGT GLGLSICRQL ScSln1p 901 : ANMMHGTMKL ESKVGVGSKF TFTLPLNQTK EI--SFADME FPFEDEFNPE SRKNRRVKF- -----_-- -----SVAKS IKSRQSTSSV ATPATNRSSL

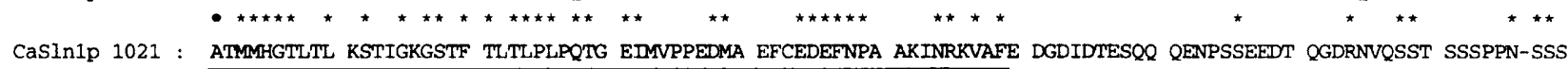
SCSln1p 983 : TNDVLPEVRS -...-KGKHE TKDVGNPNMG REEKNDNGGL EQLQEKNIK- PSICLTGAEV NEQNSLSSK- --- - - - - -.--HRSRH EGLGSVNLDR CaSln1p 1120 : TDSALPASDS SDIGGTNKSK TTSHGNNKDI NAKKRIITNS SASSTKGTKR PTNNDGGGGV NGNHSDDNKD LTLTIDKPSL FTRGSTGTAN SGTTSSHSDK SCSIn1p 1061 : PFLQSTGTAT SSRNIPTVKD DDKNETS-VK ILVVEDNHVN QEVIKRMLNL EGIENIEIAAC DGQEAFDKVK ELTSKGENYN MIFMDVQMPK VDGLISTKMI SCSIn1p $1061:$ PFLQSTGTAT SSRNIPTVKD DDKNETS-VK ILVVEDNHVN QEVIKRMLNL EGIENIEIAAC DGQEAFDKVK ELTSKGENYN MIFMDVQMPK VDGLISTKMI CaSlnIp $1220:$ KILYPTPTTT TTTTTTTDHT TVLDDISHLR VLVAEDNSVN QEVISRMLKQ EGITNLTMAC NGAKAIDFVK ESIENNENFD LIFMDVQMPE VDGLKATKMT SCSln1P 1160 : RRDLGYTSPI VALTAFADDS NIKECLESGM NGFLSKPIKR PKLKTILTEF CAAYQGKKNN K

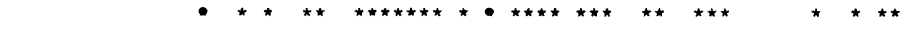
CaSln1p 1320 : RKNLOYNKPI IALTAFADES NVKECLNSGM SGFITKPISK TNIKKVLVEF ---LSNEVVT S

Fig. 2. For legend see facing page.

there are some structural differences between the two proteins. As mentioned above, N. crassa Nik1p contains six repeats of 90 aa that always start with tryptophan, but the first repeat in $\mathrm{CaNik} 1 \mathrm{p}$ begins with glutamic acid and the fourth repeat observed in the $N$. crassa Nik1p is absent from CaNik1p (Fig. 2b). The regions from amino acid residues 490 to 641 and from 886 to 990 of CaNik1p share a high degree of sequence identity with the sensor 
(b)

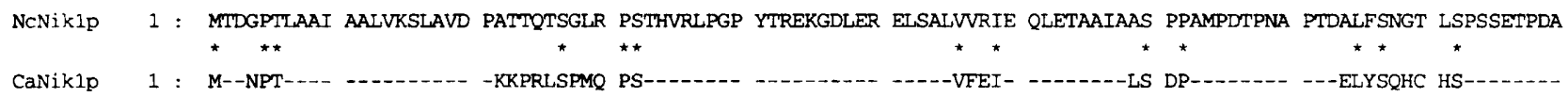

NCNik1p 101 : RYPAPLPRNG FIDEALEGLR EHVDDQSKLL DSQRQELAGV NAQLIEQKQL QEKALAIIEQ ERVATLEREL WKHQKANEAF QKALREIGEI VTAVARGDLS

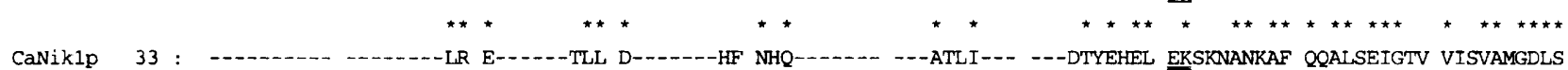

NCNik1p 201 : KKVRMNSVEM DPEITTFKRT INTMMDQLQV FSSEVSRVAR EVGTEGILGG QAQIEGVDGT WKELTDNVNV MAQNLTDQVR EIASVTTAVA HGDLTKKIER

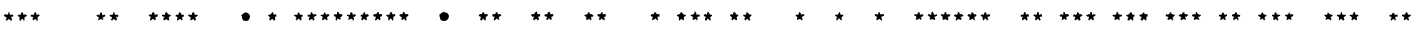

CaNik1p $86:$ KKVEIHTVEN DPEILKVKIT INTMMDQLQT FANEVTKVAT EV-ANGELGG QAKNDGSVGI WRSLTDNVNI MALNLTNQVR EIADVTRAVA KGDLSRKINV

NCNik1p 301 : PAKGEILQLQ QTINTMVDQL RTFASEVTRV ARDVGTEGIL GGQADVEGVQ GMMNELTVNV NAMANNLTTQ VRDIIKVTTA VAKGDLTQKV QAECRGEIFE

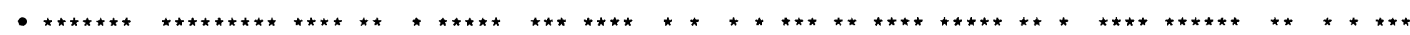

CaNik1p $185:$ HAQGEILQLQ RTINIMVDQL RTFAFEVSKV ARDVGVLGIL GGQALIENVE GIWEELTDNV NAMALNLTTQ VRNIANVTTA VAKGDLSKKV TADCKGEILD

NCNik1p $401:$ LKKTINSMVD QLQQFAREVT KIAREVGTEG RLGGQATVHD VQGTWRDLTE NVNGMAMNLT TQVRELAKVT TAVAKGDLTK KIGVEVQGEI LDLKNTINIM

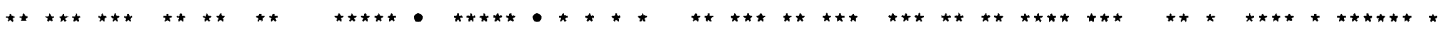

CaNik1p $285:$ LKLTINQMVD RLQNFALAVT TLSREVGTLG ILGGQANVQD VEGAWKOVTE NVNLMATNLT NQVRSIATVT TAVAHGDLSQ KIDVHAQGEI LQLKNTINKM

NCNik1P 501 : VDRLGTFAFE VSKVAREVGT DGTLGGQAQV DNVEGKWKDL TENVNTMASN LTSQVRGIST VTQAIANGDM SRKIEVEAKG EILILKETIN NMVDRLSIFC

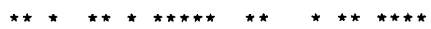

CaNiklp $385:$ VDSLQLFASE VSKVAQDVGI NGKLGIQAQV

NCNik1p 601 : NEVQKVAKDV GVDGIMGGQA DVAGLKGRWK EITTDVNTMA NNLTAQVRAF GDITNAATDG DFTKLVEVEA SGEMDEI,KKK INQMVYNLRD SIQRNTQARE

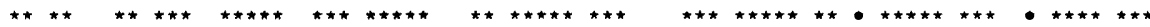
CaNik1p $415:-\ldots-\ldots$ -

NCNik1p 701 : AAEIANKTKS EFLANMSHEI RTPMNGIIGM TQLTLDTDLT QYQREMLNTV NSLANSLLTI IDDILDLSKI EARRMVIEET PYTLRGTVFN ALKTLAVKET

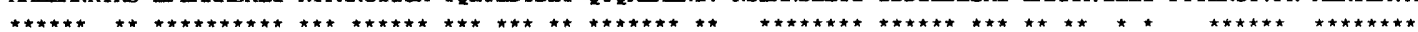

CaNik1p 493 : AAELANSAKS EFLANMSHEI RTPLNGIIGM TQLSLDTELT QYQREMLSIV HNLANSLLTI IDDILDISKI EANRMTVEQI DFSLRGTVFG ALKTLAVKAI

NCNik1p 801 : EKFLDLTYRV DHSVPDHVVG DSFRLRQIIL NLVGNAIKFT EHGEVSLTIQ KASSVQCSTE EYAIEFVVSD TGIGIPADKL DLIFDTFQQA DGSMTRKFGG

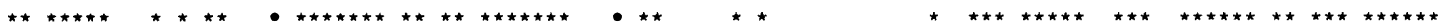
CaNik1p $593:$ EKNLDLTYQC DSSFPDNLIG DSFRLRQVIL NLAGNAIKFT KEGKVS-VSV KKSDKMVLDS KILLEVCVSD TGIGIEKDKL GLIFDTFCQA DGSTTRKFGG

NCNik1p 901 : TGLGLSISKR LVNLMGGDVW VKSEYGKGSK FFFTCVVRLA NDDISLIAKQ LNPYKSHQVL FIDKGRTGHG PEIAKML--- -HGLGLVPIV VDSERNPALE

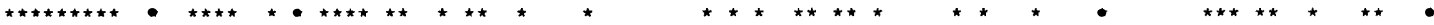
CaNik1p 692 : TGLGLSISKQ LIHIMGGEIW VTSEYGSGSN FYFTVCVSPS NIRYTRQTEQ LIPFSSHYVL F ---VSTEHT QEETDVLRDG IIELGLIPII V---RN--IE NCNik1P $997:$ KARAAGQAPY DVITVDSIED ARRLRSVDDF KYLPTVL-LA PVVHVSIKSC LDLGITSYMT TPCQLIDLGN GMVPALENRA TPSIADNTKS FEILIAEDNT

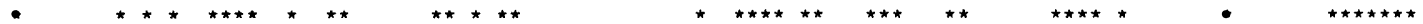
CaNik1p $784:$ DATLTEPVKY DIDMIDSIEI AKKLRLLSEV KYIPLVLVHH SIPQLNMRVC IDLGISSYAN TPCSITDLAS AIIPALESRS ISQNSDESVR YKILLAEDNL NCNik1p 1096 : VNQRIAVKIL EKYHHVVTVV GNGEEAVEAV KRKKFDVILM DVQMPIMGGF EATAKIREYE -.....-RSLG SQRTPIIALT AHAMMGREK CIQAQMDEYL CaNik1p 884 : VNQKLAVRIL EKQGHSVEVV ENGLEAYEAI KRNKYDVVLM DVQMPVMGGF EATEKIRQWE KKSNPIDSL- TFRTPIIALT AHAMLGDREK SLAKGMDDYV NCNik1p $1190:$ SKPLQQNHLI QTILKCATLG GQLLEKNRER ELTRAADAVT GGRRDNGMS ASQAAQHAAL RPPLATRGLT AADSLVSGLE SPSIVTADKE DPLSRARASL

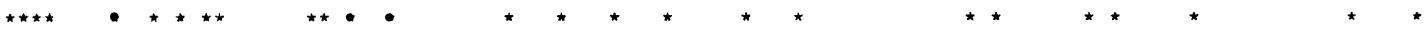
CaNik1p $983:$ SKPLKPKILM QXINKCIHNI NQLKELSRNS RGSDFAKKMT --RNTPG--- -STTRQGSDE GSVEDMIGDT PRQGSVEGGG TSS----RPV QRRSATEGSI NCNiklp $1290:$ SEPNIHKAS

CaNik1p 1073 : TTISEQIDR

Fig. 2. (a) The amino acid sequence of $S$. cerevisiae $S \ln 1 p(S c S \ln 1 p)$ is compared with that of $C$. albicans $S \ln 1 p(C a S \ln 1 p)$. Identical amino acids between $S c S I n 1 p$ and CaSIn $1 p$ are marked by asterisks. Probable transmembrane regions of ScSIn $1 p$ and CaSIn $1 \mathrm{p}$ are double-underlined. (b) The amino acid sequence of $N$. crassa Nik1p (NcNik1p) is compared with that of C. albicans Nik1p (CaNik1p). Identical amino acids between CaNik1p and NcNik1p are marked by asterisks. The first two amino acids of each repeat of about 90 aa in NcNik1p and CaNik1p are double-underlined. Amino acid sequences were aligned using the BLAST and FASTA programs. Predicted histidine kinase and receiver domains are indicated by bold underlining and dashed bold underlining, respectively. ' +' represents the positions of the histidine and aspartic acid residues that correspond to the autophosphorylated histidine and phosphorylated aspartic acid residues of ScSIn $1 \mathrm{p}$. According to the report of Santos \& Tuite (1995), the CTG codon is decoded as serine instead of leucine. 
(a)

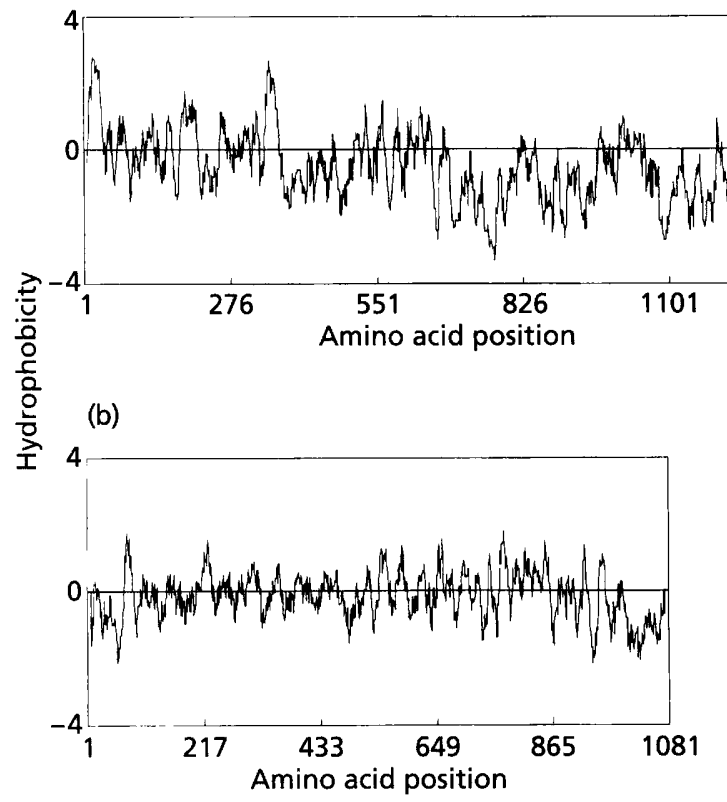

Fig. 3. Hydropathy plots of C. albicans $\operatorname{Sin} 1 p$ (a) and Nik1p (b) were calculated as described by Kyte \& Doolittle (1982) using a window of $12 \mathrm{aa}$. It should be noted that two short domains with hydrophobicity indices of above 3 in CaSIn1p (a) are considered as transmembrane helices.

kinase and response regulator domains of twocomponent systems, respectively. Sequence comparisons with $S$. cerevisiae $S \ln 1 \mathrm{p}$ and the BarA protein of Escherichia coli implicate the histidine at position 510 and the aspartic acid at position 924 as sites of phosphorylation (Fig. 2b).

\section{DISCUSSION}

We have isolated and sequenced a C. albicans homologue of ScSLN1. Like ScSln1p, CaSln1p possesses extracellular sensor, histidine kinase and receiver domains. When expressed from a multicopy plasmid, CaSLN1 overcame the growth defect of $S$. cerevisiae cells caused by the repression of $S c S L N 1$. Since CaSln1p also shares significant sequence identity with the probable ATP-binding site within the kinase domain of ScSln1p, it should be able to autophosphorylate a histidine residue of the protein.

The receiver domain of $\mathrm{CaSln} 1 \mathrm{p}$ was not necessary to rescue $\sin 1 \Delta S$. cerevisiae cells. However, the mechanism of the complementation of $\mathrm{ScS} \ln 1 \mathrm{p}$ by C-terminally truncated CaSln $1 \mathrm{p}$ is not clear. In $S$. cerevisiae, the phosphate moiety at the autophosphorylated histidine residue in the kinase domain of $\operatorname{Sin} 1 p$ is transferred to an acceptor aspartic acid residue in the receiver domain of the same protein, then to a histidine residue in the downstream Ypd1p and finally to an aspartic acid residue of the further downstream Ssk1p, with both the
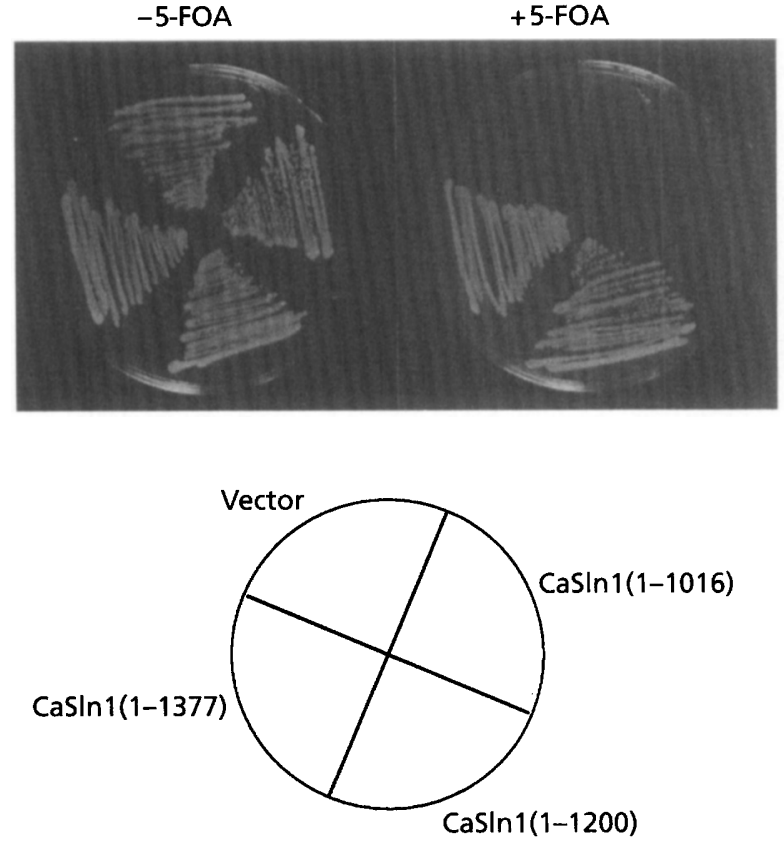

Fig. 4. Functional complementation of SCSLN1 by CaSLN1. S. cerevisiae pYES-SLN1 cells were transfected with YEp24T (vector) or YEp24T harbouring CaSLN1, which can encode full length CaSIn1p [CaSIn1(1-1377], or C-terminally truncated CaSIn $1 \mathrm{p}[\mathrm{CaS} \ln 1(1-1200)$ and CaSIn1(1-1016)]. The transfectants were spread on plates with $(+)$ or without $(-)$ 5-FOA and were incubated for $3 \mathrm{~d}$. The receiver domain is deleted in CaSln $1(1-1200)$ and the receiver domain and half of ATPbinding site are destroyed in CaSIn1(1-1016).

autophosphorylated histidine and receiver aspartic acid residues being essential for viability under low osmotic conditions (Posas et al., 1996). One possibility is that CaSln1p can bypass the phospho-relay to the receiver domain and to Ypd1p, and can function by directly phosphorylating Ssk1p.

In addition to CaSLN1, another gene, CaNIK1, was isolated and sequenced. The product of CaNIK1 is highly homologous to Nik1p of N. crassa, with regions that are highly related to the sensor kinase and response regulator domains of two-component systems. Although there is no apparent transmembrane helix in CaNik1p, we asked if CaNik1p has a similar function to ScSln1p. However, preliminary experiments did not support this hypothesis, because pYES-SLN1 cells harbouring CaNIK1 in a multicopy plasmid were unable to grow in the presence of 5-FOA or glucose. This result implies that CaNIK1 is functionally distinct from $S$. cerevisiae SLN1 and that CaSLN1 and CaNIK1 may not act in the same pathway. In fact, sequence homology between $\mathrm{CaSln} 1 \mathrm{p}$ and CaNik1p is restricted to short regions encompassing the phosphorylated histidine and receiver aspartic acid residues. However, we cannot yet rule out the trivial possibility that the CaNIK1 gene failed to function in $S$. cerevisiae.

Unexpectedly, the homozygous casln $1 \Delta$ null mutation 
(a)

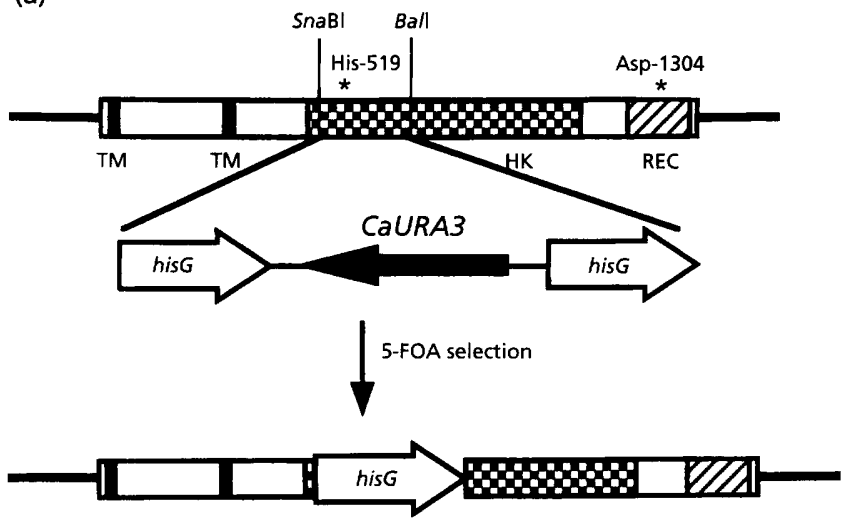

(b)

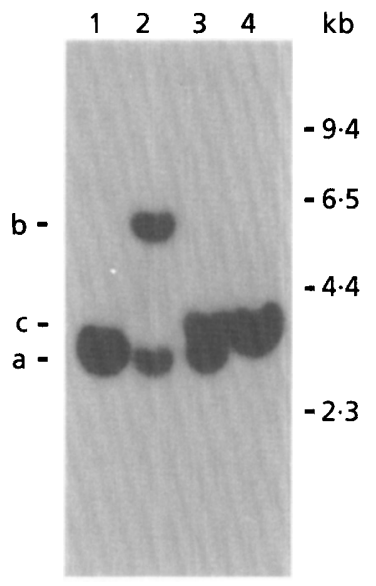

Fig. 5. Generation of the homozygous cas $\ln 1 \Delta$ null mutant strain. (a) The strategy for disrupting CaSLN1 is illustrated, with the Snal/Ball region of CaSLN1 replaced by the hisG sequences. (b) Southern blotting of CaSLN1. Twenty-five micrograms of genomic DNA from wild-type CAI4 (lane 1), the hemizygous casin1 $\triangle$ mutant with URA3 (lane 2), the hemizygous casin1 $\Delta$ mutant without URA3 (lane 3 ) and the homozygous cas $\ln 1 \Delta$ null mutant (lane 4) was digested with Bg/ll and Sall, fractionated on an agarose gel and hybridized with the $0.9 \mathrm{~kb}$ Ball-Sall fragment of CaSLN1. Bands derived from the CaSLN1 allele, the casin1 $1:$ :hisG-URA3-hisG allele and the cas $\ln 1 \Delta::$ his $G$ allele are indicated by $a, b$ and $c$, respectively.

was not lethal in C. albicans. The homozygous cas $\ln 1 \Delta$ null mutant cells grew even under high osmotic conditions, but growth in the presence of $1.5 \mathrm{M} \mathrm{NaCl}$ was somewhat impaired by disruption of CaSLN1. This phenotype resembles that of the nik1 $1 \Delta$ null mutant of $N$. crassa (Alex et al., 1996) and also that of the hog1s null mutant of C. albicans (Jose et al., 1996) and is suggestive of a CaSln $1 \mathrm{p}$ function under high osmotic conditions. In contrast, the histidine kinase activity of $S c S \ln 1 \mathrm{p}$ is necessary under low osmotic conditions and is repressed under high osmotic conditions, leading to activation of the HOG1 MAP kinase in S. cerevisiae. Thus, it seems to be puzzling why a disruption of casln $1 \Delta$ caused a growth defect at high osmolarity. Although further experiments, including the disruption of CaNIK1, are under way to (a)
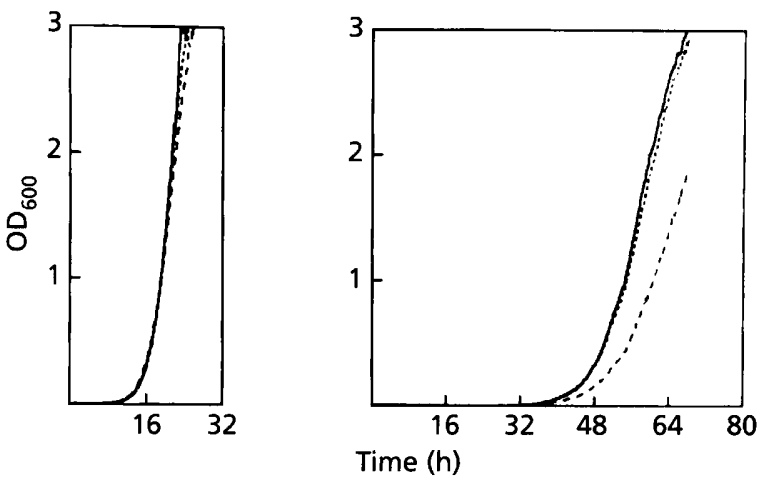

(b)

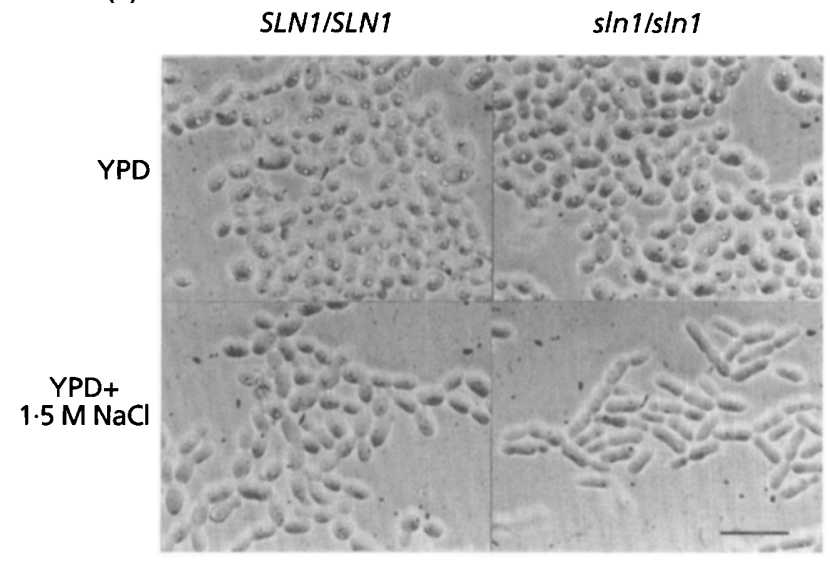

Fig. 6. Effects of the disruption of CaSLN1 on growth and morphology. (a) Effects of high osmolarity on the growth of wild-type CAl4 (SLN1/SLN1) (-), the hemizygous casin1 $\Delta$ mutant $(S L N 1 / \sin 1 \Delta)(\ldots)$ and the homozygous cas $\ln 1 \Delta$ null mutant $(s \ln 1 \Delta / s \ln 1 \Delta)(---)$. Cells of the indicated strains were cultured in YPD medium in the absence (left) or presence (right) of $1.5 \mathrm{M} \mathrm{NaCl}$ and the growth of the cells was monitored using a Biophotorecorder (Advantec). (b) Morphological change caused by the disruption of CaSLN1. Cells of wild-type CAl4 (SLN1/SLN1) and of the homozygous $\sin 1 \Delta$ null mutant $(\sin 1 \Delta / \sin 1 \Delta)$ were cultured in YPD medium in the absence (upper panels) or presence (lower panels) of $1.5 \mathrm{M} \mathrm{NaCl}$. Photographs of cells from overnight cultures are shown. Bar, $10 \mu \mathrm{m}$.

address this question, the absence of a NIK1 homologue in the $S$. cerevisiae genome suggests a divergence of osmosensing signal transduction mechanisms in fungi.

\section{ACKNOWLEDGEMENTS}

We thank B. L. Schneider for pMPY-ZAP, and S. Veronneau and A.-M. Sdicu for DNA sequencing. This work is supported in part by grant from the Ministry of Education and Science, Japan to T.O. Shigehisa Nagahashi and Toshiyuki Mio contributed equally to this work.

\section{REFERENCES}

Alex, L. A., Borkovich, K. A. \& Simon, M. I. (1996). Hyphal development in Neurospora crassa: Involvement of a two- 
component histidine kinase. Proc Natl Acad Sci USA 93, 3416-3421.

Baudin, A., Ozier-Kalageropoulos, O., Denouel, A., Lacroute, F. \& Culin, C. (1993). A simple and efficient method for direct gene deletion in Saccharomyces cerevisiae. Nucleic Acids Res 21, 3329-3330.

Bourret, R. B., Borkovich, K. A. \& Simon, M. I. (1991). Signal transduction pathways involving protein phosphorylation in prokaryotes. Annu Rev Biochem 60, 401-441.

Brewster, J. L., de Valoir, T., Dwyer, D., Winter, E. \& Gustin, M. C. (1993). An osmosensing signal transduction pathway in yeast. Science 259, 1760-1763.

Brown, J. L., North, S. \& Bussey, H. (1993). SKN7, a yeast multicopy suppressor of a mutation affecting cell wall $\beta$-glucan assembly, encodes a product with domains homologous to prokaryotic two-component regulators and to heat shock transcription factors. J Bacteriol 175, 6908-6915.

Chang, C., Kwok, S. F., Bleecker, A. B. \& Meyerowitz, E. M. (1993). Arabidopsis ethylene-response gene ETR1: similarity of product to two component regulators. Science 262, 539-544.

Fonzi, W. A. \& Irwin, M. Y. (1993). Isogenic strain construction and gene mapping in Candida albicans. Genetics 134, 717-728.

Hua, J., Chang, C., Sun, Q. \& Meyerowitz, E. M. (1995). Ethylene insensitivity conferred by Arabidopsis ERS gene. Science 269, 1712.

Jose, C. S., Monge, R. A., Perez-Diaz, R., Pla, J. \& Nombela, C. (1996). The mitogen-activated protein kinase homolog HOG1 gene controls glycerol accumulation in the pathogenic fungus Candida albicans. J Bacteriol 178, 5850-5852.

Kyte, J. \& Doolittle, R. F. (1982). A simple method for displaying the hydrophobic character of a protein. J Mol Biol 157, 105132.

Maeda, T., Wurgler-Murphy, S. M. \& Saito, H. (1994). A two component system that regulates an osmosensing MAP kinase cascade in yeast. Nature 369, 242-245.

Mio, T., Yabe, T., Sudoh, M., Satoh, Y., Nakajima, T., Arisawa, M. \& Yamada-Okabe, H. (1996). Role of three chitin synthase genes in the growth of Candida albicans. J Bacteriol 178, 2416-2419.
Nagahashi, S., Nakayama, H., Hamada, K., Yang, H., Arisawa, M. \& Kitada, K. (1997). Regulation by tetracycline of gene expression in Saccharomyces cerevisiae. Mol Gen Genet 255, 372-375.

Ota, I. M. \& Varshavsky, A. (1993). A yeast protein similar to bacterial two component regulators. Science 262, 566-569.

Parkinson, J. S. \& Kofoid, E. C. (1992). Communication modules in bacterial signaling proteins. Annu Rev Genet 26, 71-112.

Posas, F., Wurgler-Murphy, S. M., Maeda, T., Witten, E., Thai, T. C. \& Saito, H. (1996). Yeast HOG1 MAP kinase cascade is regulated by a multiple phosphorylating mechanism in the SLN1-YPD1-SSK1 'two-component' osmosensor. Cell 86, $865-875$.

Sambrook, J., Fritsch, E. F. \& Maniatis, T. (1989). Molecular Cloning: a Laboratory Manual, 2nd edn. Cold Spring Harbor, NY: Cold Spring Harbor Laboratory.

Sanglard, M., Ischer, F., Monod, M. \& Bille, J. (1997). Cloning of Candida albicans genes conferring resistance to azole antifungal agents: characterization of $C D R 2$, a new multidrug $\mathrm{ABC}$ transporter gene. Microbiology 143, 405-416.

Santos, M. A. S. \& Tuite, M. F. (1995). The CUG codon is decoded in vivo as serine and not leucine in Candida albicans. Nucleic Acids Res 23, 1481-1486.

Schneider, B. L., Steiner, B. T., Seufert, W. \& Futcher, A. B. (1996). pMPY-ZAP: a reusable polymerase chain reaction-directed gene disruption cassette for Saccharomyces cerevisiae. Yeast 12, 129-134.

Stock, J. B., Lukat, G. S. \& Stoch, A. M. (1991). Bacterial chemotaxis and the molecular logic of intracellular signal transduction networks. Annu Rev Biophys Chem 20, 109-136.

Wilkinson, J. Q., Lanahan, M. B., Yen, H.-C., Giovannoni, J. J. \& Klee, H. J. (1995). An ethylene-inducible component of signal transduction encoded by Never-ripe. Science 270, 1807-1809.

Yamada-Okabe, T., Shimmi, O., Doi, R., Mizumoto, K., Arisawa, M. \& Yamada-Okabe, H. (1996). Isolation of the mRNA-capping enzyme and ferric-reductase-related genes from Candida albicans. Microbiology 142, 2515-2523.

Received 19 August 1997; revised 14 October 1997; accepted 20 October 1997. 